\title{
High-Field MRI Contribution in Tolosa-Hunt Syndrome (THS). Report of Two Cases and Review of Literature
}

\author{
Chérif Mohamadou Aidara1 ${ }^{*}$, Philomène Kouna², Jennifer Nyangui Mapaga², Nfally Badji1, \\ Hamidou Deme1, Abdoulaye Dione Diop' ${ }^{1}$, Abdoulaye Ndoye Diop${ }^{1}$, Sokhna Ba' ${ }^{1}$ El Hadj Niang ${ }^{1}$ \\ ${ }^{1}$ Radiology Department, Fann University Hospital, Dakar, Sénégal \\ ${ }^{2}$ Neurology Department, El Rapha Polyclinic Trois Quartiers BP 256, Libreville, Gabon \\ Email: *matoouz@gmail.com, *cfm.aidara@univ-zig.sn
}

How to cite this paper: Aidara, C.M., Kouna, P., Mapaga, J.N., Badji, N., Deme, H., Diop, A.D., Diop, A.N., Ba, S. and Niang, E.H. (2018) High-Field MRI Contribution in Tolosa-Hunt Syndrome (THS). Report of Two Cases and Review of Literature. Open Journal of Radiology, $\mathbf{8}$, 141-149.

https://doi.org/10.4236/ojrad.2018.83016

Received: April 14, 2018

Accepted: July 20, 2018

Published: July 23, 2018

Copyright $\odot 2018$ by authors and Scientific Research Publishing Inc. This work is licensed under the Creative Commons Attribution International License (CC BY 4.0).

http://creativecommons.org/licenses/by/4.0/

\section{(c) (i) Open Access}

\begin{abstract}
Background: Tolosa Hunt Syndrome is an inflammatory condition of unknown etiology of the cavernous sinus and superior orbital fissure. Because of the difficulty in establishing histological evidence, his diagnosis is based on a set of arguments established by the International Headache Society. MRI allows indirect visualization of the granuloma and plays a key role in diagnosis and follow-up. Aim: To illustrate High-field MRI contribution in Tolosa-Hunt Syndrome (THS). Cases Presentation: Two patients, a 25-year-old female and a 40-year-old male were recruited in this retrospective case report study at the Radiology service of Fann University Hospital (Dakar Senegal). The first patient has been suffering from a right retro-orbital pain and diplopia for 2 months and the second from a painful oculomotor nerve palsy for 3 months. Blood tests, lumbar puncture, thyroid hormone levels and an infectious screen were done. Screening for converting enzymes, and serum antibodies were also done. They underwent a high field MRI (Siemens 1.5T) with T1, T2, FLAIR, T2* diffusion B1000, TOF polygon, CISS 3D and T1 gadolinium sequences in the 3 planes space. No significant abnormality was detected in blood tests or CSF analysis. Screening for converting enzymes and serum antibodies screen were also negative. For each patient, MRI examinations showed a non tumoral thickening of the right cavernous sinus, suggesting a granulomatous involvement. Tolosa Hunt Syndrome was evoked firstly. They were put on corticotherapy at high doses with a spectacular regression of symptoms. The Criteria of the International Headache Society of THS were met in both patients. Conclusion: High-field MRI is a significant diagnostic tool in the assessment of painful ophthalmoplegia. It allows a direct visualization of the granuloma of the cavernous sinus and assesses its course throughout the disease.
\end{abstract}




\section{Keywords}

Painful Ophthalmoplegia, Tolosa-Hunt Syndrome, Criterias of International Headache Society, High-Field MRI

\section{Introduction}

Tolosa Hunt Syndrome (THS) is an inflammatory granulomatosis of unknown etiology, with a chronic course and which involves the cavernous sinus and the superior orbital fissure. It's a rare condition before the age of 20 and it mainly affects young adults. Diagnosis of THS is difficult as evidenced by the evolution of the criteria of the International Headache Society (IHS). These criteria highlight all the difficulty of establishing evidence based diagnosis.

Diagnosis key of THS is based on the histological evidence of inflammatory granuloma. Only MRI currently allows visualizing it indirectly, which highlights the role of MRI in this pathology.

The cavernous sinus is deep and not easy to explore. Since the initial description by Tolosa, exploration techniques have been significantly improved; this condition is well known nowadays because of advances in medical imaging. High-field MRI especially, has an optimal soft tissue resolution to study the vessels, nerves and meningeal structures of this close part of the skull base. We want to discuss through two clinical cases, the clinical findings and MRI contribution of TSH whose treatment is mainly based on corticosteroid therapy.

\section{Clinical Cases}

\subsection{Case 1}

Mrs AL, 25 years old, with no relevant medical history, had a right retro-orbital pain and diplopia for 2 months. The pain is partially relieved with analgesics such as paracetamol. On examination there was right oculomotor nerve palsy with ptosis and external strabismus. Blood tests (Full blood count, CRP), lumbar puncture, thyroid hormone levels and an infectious screen (retroviral serology, syphilis, hepatitis B and C) were negative. Screening for converting enzymes, and serum antibodies ANCA, native anti-SM, antinuclear, anti-SSB, anti-DNA were also negative. She was sent for an MRI assessment.

- MRI features

She had undergone a high field MRI (siemens 1.5T) with T1, T2, FLAIR, T2*, diffusion B1000, TOF polygon, CISS 3D and T1 gadolinium sequences in the 3 space planes. Exploration had found an enlarged right cavernous sinus, with a soft tissue infiltration that appears isosignal T2 SE on Figure 1(a). Its involvement is compared to the left side, which is normal. The lateral wall of the sinus appeared hyposignal and was well identified on the same figure. Global morphology of the cavernous sinus and the Meckel's cavum were respected as showed on Figure 1(c). The lesion was strongly enhanced after gadolinium 


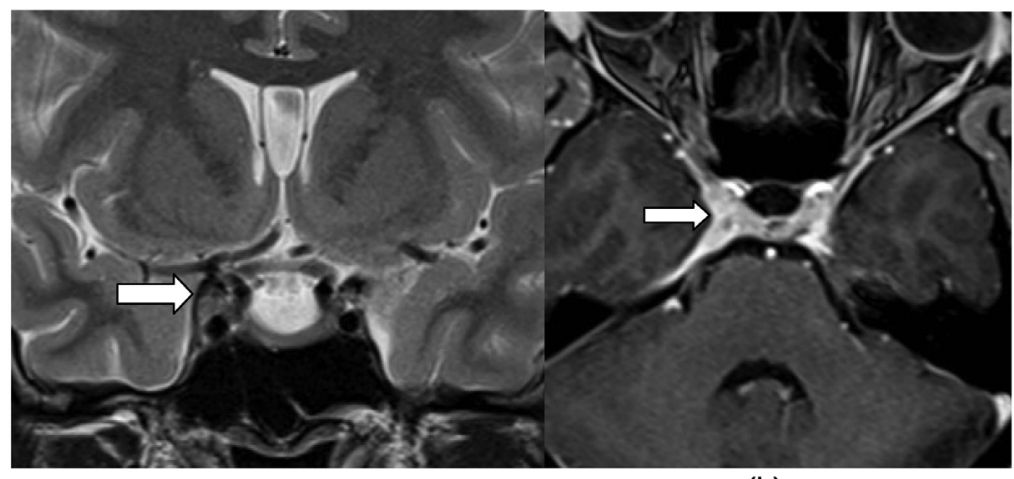

(a)

(b)

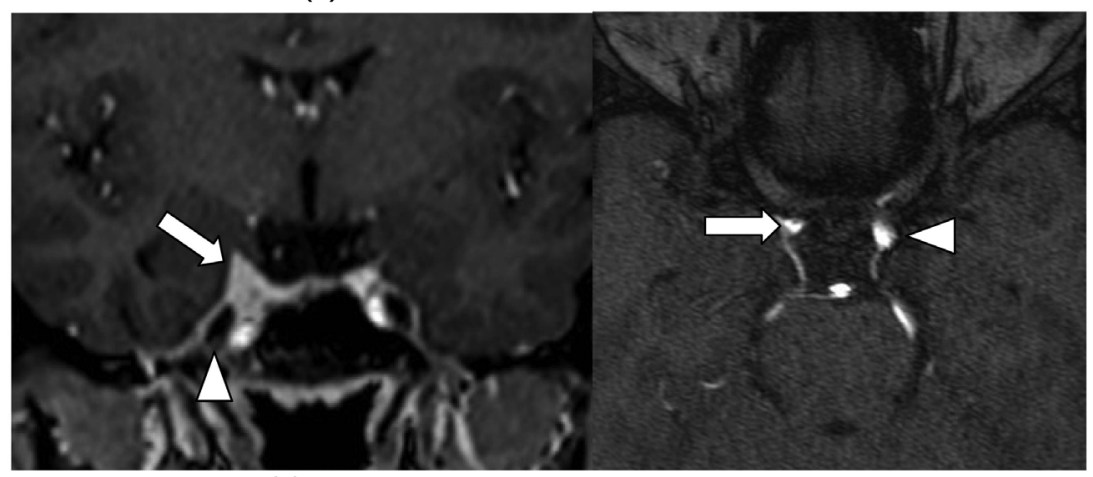

(c)

(d)

Figure 1. Coronal T2 SE (a) showing an isointense thickening of the right cavernous sinus (arrow (a)) compared to the left normal (arrowhead (b)); Axial T1 gadolinium (b) showing strong diffuse contrast enhancement of the right cavernous sinus without (arrow (b) and (c)); Coronal section T1 after gadolinium injection, showing the lesion that let intact the Meckel's cavity (arrowhead (c)); TOF axial native section (d) showing a significant mass effect on the right internal carotid (arrow (d)) compared to the left internal carotid (arrowhead $(\mathrm{d}))$.

injection on Figure 1(b) and Figure 1(c). There was also a slight compression of the intracavernous ipsilateral internal carotid on Figure 1(d). These aspects pleaded for a diffuse inflammatory involvement.

- Presumptive diagnosis: Tolosa Hunt Syndrome

- Course under corticotherapy

She received a daily dose of $60 \mathrm{mg}$ intravenous solumedrol for 3 days, followed by an oral relay (prednisolone $60 \mathrm{mg} /$ day). There was a significant but not complete regression of symptoms in 72 hours for the pain and the paralysis of the oculomotor nerve. At 10 days, the symptoms had regressed almost completely. At one month of treatment regression of pain and oculomotor nerve involvement were complete.

After an 18 months' clinical follow-up with no painful recurrence or involvement of the cranial nerves, the patient was lost of sight.

- Final diagnosis: Tolosa Hunt Syndrome

\subsection{Case 2}

Mr SK, 40 years old, with no relevant medical history, presented a painful ocu- 
lomotor nerve palsy since 3 months. For him also, the blood tests, the immunological and CSF examination showed no significant abnormalities.

- MRI features

He underwent a MRI examination with the same sequences. It showed an enlargement of the right cavernous sinus compared to the left side. The cavernous sinus appeared isosignal T2 in Figure 2(a). There was a significant gadolinium enhancement after injection. The enhancement extended to the superior orbital fissure and the adjacent pachymeningeal structures as showed in Figure 2(b). The sagittal MIP reconstructions of the TOF sequence showed a slight reduction of the right internal carotid lumen on Figure 2(c) compared to the left side on Figure 2(d). These aspects pleaded, here again for an inflammatory involvement.

- Presumptive diagnosis: Tolosa Hunt Syndrome

- Course under corticotherapy

He also received $60 \mathrm{mg}$ intravenous solumedrol for 3 days followed by an oral relay (prednisolone $60 \mathrm{mg}$ /day). The regression of symptoms was also spectacular. In 3 days there was a clear regression of the pain, at one week the paralysis had regressed partially but significantly and at one month the recovery was complete.

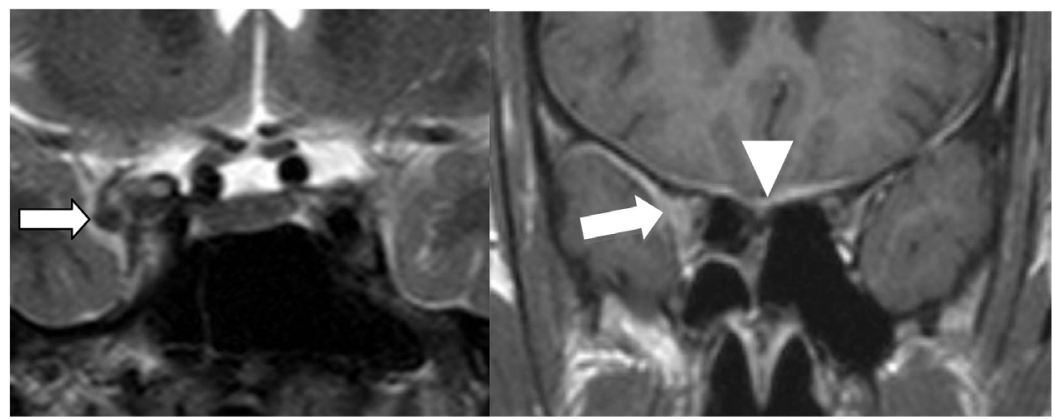

(a)

(b)

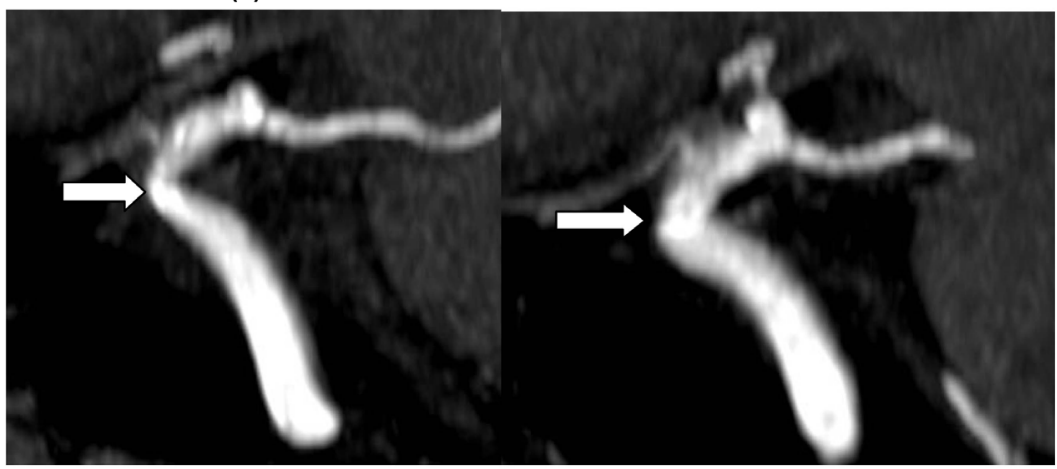

(c)

(d)

Figure 2. The coronal T2 image (a) shows an enlarged isosignal T2 in the right cavernous sinus significantly enhanced after gadolinium injection (arrow (a) and (b)). The image on (b) shows the pachymeningeal contrast enhancement (arrow-head (b)). The sagittal 3D TOF MIP reconstruction (c) shows a slightly decrease lumen diameter of the right internal carotid (arrow (c)) due to inflammation (arteritis) or a slight compression compared to the normal left internal carotid lumen diameter (arrow (d)). 
Clinical follow-up was established for 12 months. There was no pain recurrence or involvement of the cranial nerves.

- Final diagnosis: Tolosa Hunt syndrome

\section{Comments}

\subsection{HIS Criteria of THS}

THS is a painful inflammatory ophthalmoplegia, most often unilateral. Its etiology is unknown. Tolosa reported in 1954 the first case in a patient with left orbital pain associated with a progressive decrease of visual acuity, ophthalmoplegia and hypoesthesia in the ophthalmic nerve area. Carotid angiography demonstrated a narrowing of the intracavernous internal carotid artery. Autopsy revealed granulomatosis inflammation of the left internal carotid and cavernous sinus. In 1961 Hunt described 6 similar cases. It was in 1966 that Smith and Taxdal gave this pathological entity the name of Tolosa-Hunt Syndrome [1] [2].

The IHS classifies TSH as part of episodic orbital pain associated with third, fourth and/or sixth cranial nerve palsy that resolves spontaneously with a tendency to relapse. Its diagnostic criteria are below:

- One or more episodes of unilateral orbital pain lasting for weeks if not treated,

- Paresis of one or more of the third, fourth or sixth cranial nerve and/or the demonstration of granuloma by MRI or biopsy,

- Paresis coincides with pain or follows it within 2 weeks,

- Pain and paresis resolve within 72 hours when adequately treated by corticosteroids,

- Other causes of ophthalmoplegia are excluded with the appropriate investigations (tumors, vasculitis, basal meningitis, sarcoidosis, diabetes mellitus and ophthalmoplegic migraine).

The IHS outlined however, that some reported cases of THS are associated with sympathetic, ophthalmic nerve, optic nerve, or acoustico-facial bundle involvement and that the careful monitoring is required to rule out other causes of painful ophthalmoplegia [1] [3] [4]. These IHS criteria's emphasize the need to demonstrate the inflammatory granuloma by MRI or biopsy on the one hand and to rule out another cause of painful ophthalmoplegia by the appropriate examinations. High field MRI is the most powerful current technique to explore the cavernous sinus, the superior orbital fissure and the skull base. With its high soft tissue resolution, its multiform contrast and the possibility of tridimensional study, it gives such information to evoke the diagnosis of THS and to rule out other causes of painful ophthalmoplegia.

\subsection{MRI Semiology}

Involvement of the cavernous sinus and the superior orbital fissure in the THS appears as a diffuse infiltration isosignal on T2 weighted sequence with enlargement of the cavernous sinus [Figure 1 and Figure 2] [5] [6] [7] [8] [9]. After gadolinium injection, there is a diffuse and significant enhancement that 
reaches the neighboring regions such as the orbital apex or the superior orbital fissure. Sometimes it is associated with a contrast enhancement of the adjacent pachymeningeal structures as noted on Figure 2.

Carotid involvement is part of the original description of Tolosa. It seems to be a characteristic [3] [4] [5] [6] that can be related to compression by the granuloma and/or vasculitis substantially reducing the carotid lumen as observed in our patients [Figure 1 and Figure 2]. Carotid involvement seems not to have any major encephalic consequence such as ischemia throughout literature. So there was in our patients.

The particularities of our observations lie in the fact of being able to demonstrate the diffuse inflammatory involvement of the cavernous sinus, the involvement of the adjacent pachymeningeal structures and the intra-cavernous internal carotid. These aspects are the keys to the diagnosis of this pathology as illustrated by the IHS criteria.

It should be noted that the semiology of cavernous inflammatory disease is not specific to the THS, which is a limit of this examination [3]. Tumor origin, granulomatous origin (sarcoidosis, Wegener's disease), lymphomatous disease, vascular diseases are not excluded. A nasopharyngeal tumor can be easily excluded by routine scanning of the nasopharynx during MRI examination. In a personal unpublished serie of oculomotor nerve palsy explored by MRI, a case of nasopharyngeal tumor was discovered fortuitously as illustrated on Figure 3. One should need to systematically scan the nasopharyngeal region during imaging examination of the common oculomotor nerve.

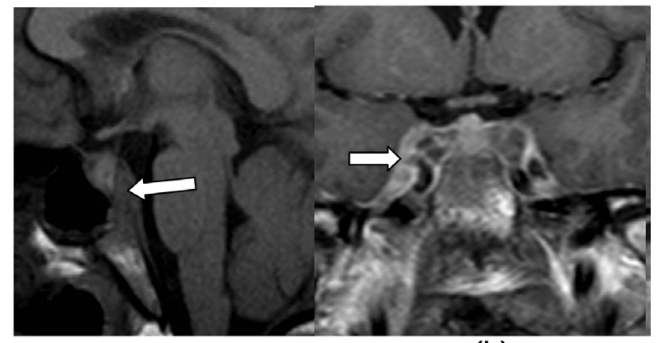

(a)

(b)

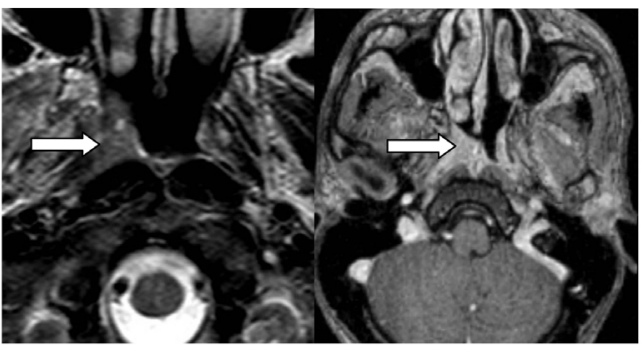

(c)

(d)

Figure 3. Sagittal section T1SE in (a) focused on the clivus showing a bone marrow replacement suspected of metastasis (arrow on (a)). The T1 coronal section gadolinium on (b) shows a thickening with contrast enhancement of the right cavernous sinus (arrow). The T2SE axial section on (c) and that in T1 gadolinium on (d) show a tumoral filling of the right Rosenmüller fossa strongly enhanced after gadolinium injection (arrow (c) and (d)). 
In the cases reported by Shama, et al., the presence of nodule or bone lesion was associated with metastatic disease or lymphoma [8]. That's why IHS and many authors advise rigorous monitoring to rule out another cause of painful ophthalmoplegia. Shama, et al. proposed a minimum 6 weeks' follow-up while Mandrioli and La Mantia proposed 2 years. It should be emphasized in the follow-up the interest of MRI before and after corticosteroid therapy [5] [10]. Gimenez-Roldan, et al. reported a 13-years-followed-case [11].

Mandrioli reported a THS case due to an actinomycosis revealed during the follow-up. This highlights the importance of clinical monitoring and imaging in management. The biopsy of this region is not without danger and must be considered as a last resort [10].

However, MRI may be normal during Tolosa Hunt syndrome. In the series of Chih-Hseien, et al., causes of painful ophthalmoplegia with normal MRI (examination with gadolinium injection) were ocular diabetic neuropathy (44.8\%), THS (46.6\%) or ophthalmoplegic migraine (8.6\%) [12].

It is necessary for MRI to be performed with injection of gadolinium as a response to "La Mantia". Tumor exclusion includes absence of nodular enhancement or metastatic bone lesion as seen on Figure 3 [4] [8].

\subsection{Patho-Anatomical Findings in THS}

THS involvement consists histologically of a non-specific inflammatory chronic granuloma with fibroblastic and lymphocytic proliferation of the cavernous sinus wall [3]. This initial Tolosa's description was confirmed by Hunt. No infectious cause was noted on the initial description. Its etiology remains unknown.

Should these original data of this syndrome be preserved for history or should they evolve? Explorations are nowadays significantly better improved than during Tolosa and Hunt's time. Can THS be considered as an inflammatory cavernous sinus syndrome for which etiological research needs to be specified? It is in this last way that Mandrioli seems to go when describing THS due to actinomycosis parasite [10]. Contrary to the opinion of most of the authors who remain attached to the previous data discribed by Tolosa and Hunt.

\subsection{Course of THS}

THS can spontaneously regress. High doses of corticosteroids (one of criterias of the IHS) are spectacular but relapses are also classic, sometimes even in the contra-lateral side [13]. This spectacular evolution under corticosteroid is a limit not to be ignored because inflammatory pathologies in general (sarcoidosis, Wegener granulomatosis) or tumours (lymphoma) can respond favorably to corticotherapy [3]. This highlights the importance of monitoring as recommended by the IHS, and to emphasize again that THS remains a diagnosis of exclusion.

\section{Conclusion}

IHS criterias of THS significantly show the MRI contribution in the manage- 
ment of this condition throughout the course of the disease. However, limits of MRI examination lie on three facts. Firstly, what to do with a normal MRI for a significant clinical signs on the one hand. Secondly, if MRI shows an inflammatory granuloma appearance, it cannot predict its etiology. Thirdly, many inflammatory diseases respond positively to corticosteroid therapy. Only a careful follow-up seems relevant in order to rule out another cause. MRI examinations should be with gadolinium injection to compare sequences between them efficiently.

\section{Conflict of Interest}

No.

\section{Bibliography}

[1] International Headache Society (2016) Criteria for Tolosa Hunt Syndrome. IHS13.16 ICD-10 G44.850. http://ihs-classification.org/en/02_klassifikation/04_teil3/13.16.00_facialpain.html

[2] Alioglu, Z., Akbas, A., Sari, A., Erdöl, H. and Özmenoglu, M. (1999) Tolosa Hunt Syndrome: A Case Report. Journal of Neuroradiology, 26, 68-72.

[3] Kline, L.B. and Hoyt, W.F. (2001) The Tolosa-Hunt Syndrome. Journal of Neurology, Neurosurgery \& Psychiatry, 71, 577-582. https://doi.org/10.1136/jnnp.71.5.577

[4] La Mantia, L., Curone, M., Rapoport, A.M. and Bussone, G. (2006) Tolosa-Hunt Syndrome: Critical littéRature Review Based on IHS 2004 Criteria. Cephalalgia, 26, 772-781. https://doi.org/10.1111/j.1468-2982.2006.01115.x

[5] Çakirer, S. (2003) MRI Findings in Tolosa-Hunt Syndrome before and after Systemic Corticosteroid Therapy. European Journal of Radiology, 45, 83-90. https://doi.org/10.1016/S0720-048X(02)00012-8

[6] Desai, S.P., Carter, J. and Jinkins, J.R. (1991) Contrast-Enhanced MR Imaging of Tolosa-Hunt Syndrome: A Case Report. American Journal of Neuroradiology, 12, 182-183.

[7] Jain, R., Sawhney, S., Koul, R.L. and Chand, P. (2008) Tolosa-Hunt Syndrome: MRI Appearances. Journal of Medical Imaging and Radiation Oncology, 52, 447-451. https://doi.org/10.1111/j.1440-1673.2008.01988.x

[8] Shama, S.A. and Gheida, U. (2012) Superior Orbital Fissure Syndrome and Its Mimics: What the Radiologist Should Know? The Egyptian Journal of Radiology and Nuclear Medicine, 43, 589-594.

[9] Apkinar, C.K., Ozbenli, T., Dogru, H. and Incesu, L. (2017) Tolosa-Hunt Syndrome. Cranial Neuroimaging Findings. Arch Neuropsychiatry, 54, 251-254. https://doi.org/10.5152/npa.2016.13791

[10] Mandrioli, J., Frank, G., Sola, P., Leone, M.E., Guaraldi, G., Guaraldi, P., Collina, G., Roncaroli, F. and Cortelli, P. (2004) Tolosa-Hunt Syndrome Due to Actinomycosis of the Cavernous Sinus: The Infectious Hypothesis Revisited. Headache, 44, 806-811. https://doi.org/10.1111/j.1526-4610.2004.04149.x

[11] Gimenez-Roldan, S., Guillem, A. and Munoz, L. (2006) Long-Term Risk of Relapses in Tolosa-Hunt Syndrome. Neurologia, 21, 382-385.

[12] Hung, C.-H., Chang, K.-H., Chu, C.-C., Liao, M.-F., Chang, H.-S., Lyu, R.-K., et al. (2014) Painful Ophthalmoplegia with Normal Cranial Imaging. BMC Neurology, 
14, 7. http://www.biomedcentral.com/1471-2377/14/7 https://doi.org/10.1186/1471-2377-14-7

[13] Navi, B.B. and Safdieh, J.E. (2010) Recurrent, Alternating Tolosa-Hunt Syndrome. The Neurologist, 16, 54-55. https://doi.org/10.1097/NRL.0b013e3181a0ae21 